\title{
Exploring human gastric cancers through organoid culture
}

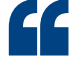 \\ ...the genetic complexity of gastric cancers has hindered the linkage of these subtypes to biological phenotypes...}

A new study published in Cell details the efficient generation and characterization of human gastric cancer organoids derived from patients with different tumour subtypes. The new findings clarify key pathways involved in gastric carcinogenesis, including the different mechanisms by which cancers gain WNT signalling independence.

Gastric cancer is a leading cause of global cancer deaths. A highly heterogeneous disease, sequencing analyses conducted in the past few years have identified four genetic tumour subtypes: genomically stable; Epstein-Barr virus (EBV)positive; microsatellite-unstable; and chromosomally unstable. However, the genetic complexity of gastric cancers has hindered the linkage of these subtypes to biological phenotypes, such as metastatic potential, growth factor dependency or anticancer drug susceptibility. "To phenotypically interpret genetic mutations identified in sequence studies, researchers have commonly used genetic mouse models," explains author Toshiro Sato. "We realized that most knockout mouse models for gastric cancers employed clinically irrelevant mutations, rendering it difficult to translate their phenotypes to clinics."

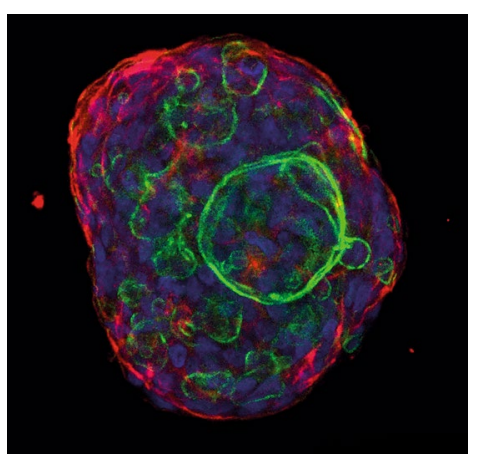

Human gastric cancer organoid. Image courtesy of T. Sato, Keio University, Japan
To address this modelling problem, Sato and colleagues turned to organoid culture systems in which a specific combination of growth factors are used to sustain the self-renewal of gastric epithelial stem cells derived from primary tissue. The first step was to optimize culture conditions to selectively grow patient-derived organoids from cancer cells, rather than the normal cells that contaminate patient tissue samples. By selecting cancer organoids using modulators of pathways commonly dysregulated in gastric cancer, the researchers improved organoid establishment efficiency from $55 \%$ to $75 \%$. In total, sequencing analysis confirmed that 36 individual patient-derived gastric cancer organoids were established: seven from microsatellite-unstable tumours; 25 from chromosomally unstable tumours; and four from genomically stable tumours. No EBV-positive tumour organoids were derived because of their rarity in the patient cohort. Each organoid culture then underwent comprehensive genetic, transcriptomic, epigenetic and morphological characterization.

One key feature of the established organoids was the high prevalence of WNT and R-spondin signalling independence. Of the 36 gastric cancer organoids, 12 grew fully independently of endogenous or exogenous WNT ligands. Functional experiments revealed that this WNT signalling independence was achieved through a variety of mechanisms, including epigenetic regulation and loss of APC protein function. Of the 24 gastric cancer organoids dependent on WNT ligands, 15 were able to grow independently of R-spondin. "From previous findings, this phenotype could be only explained by two types of molecular alterations:
R-spondin-overexpressing gene fusions or RNF43/ZNRF3 mutations," says Sato. Analysis showed that five organoids harboured alterations to RNF43 or ZNRF3, yet the majority achieved R-spondin independence via alternative pathways. To identify the responsible changes, the investigators conducted an unbiased mutation screen using sequencing data from R-spondin-independent and R-spondin-dependent organoids. Simultaneous mutations in $\mathrm{CDH} 1$ and TP53 were found in seven R-spondinindependent cultures. "An advantage of the organoid system is that we can immediately validate the screening result by CRISPR-based prospective gene engineering," comments Sato. "Indeed, we confirmed that CDH1-TP53 compound mutations conferred R-spondin independency in CRISPR-engineered normal gastric organoids."

The authors note that the findings of this study should support the ongoing development of smallmolecule WNT signalling inhibitors, which are currently being tested for their use in various cancers. Moreover, they argue that the efficient organoid derivation system used in the paper offers opportunities for studying rare gastric cancer subtypes. "Conducting a clinical trial targeting cancers with rare subtypes is not feasible at present," Sato notes. "By capturing rare cancers as living organoids, we envision that a 'clinical trial in a dish, or 'organoid-based medicine', will open up a new avenue for developing novel therapeutic strategies to treat rare subtype cancers."

Hugh Thomas

ORIGINAL ARTICLE Nanki, K. et al. Divergent routes toward Wnt and R-spondin niche independency during human gastric carcinogenesis. Cell 174, 856-869 (2018) 